\title{
La colonoscopía virtual podría ser eficaz para el rastreo de cáncer de colon
}

\section{Objetivo}

Evaluar las características operativas de la Colonoscopía Virtual (CV) y establecer una comparación con la Colonoscopía Optica (CO) convencional para el screening de cáncer de colon.

\section{Diseño}

Estudio de corte transversal.

\section{Lugar}

3 centros médicos de EEUU (National Naval Medical Center).

\section{Pacientes}

1233 adultos asintomáticos de 50 a 79 años con riesgo habitual de cáncer de colon. Se excluyeron pacientes con test positivo para sangre oculta en materia fecal, anemia, hemorragia digestiva, pérdida de peso involuntaria, colonoscopía óptica o colon por enema recientes, antecedentes de pólipos colónicos, cáncer de colon, enfermedad inflamatoria intestinal, o síndromes de poliposis familiar.

\section{Descripción de los tests y del gold Standard}

Después de la preparación colónica las 24 horas previas y previa insuflación, se realizó la tomografía computada colónica con tomógrafos de alta resolución y se realizó la reconstrucción tridimensional del colon por sustracción digital, permitiendo así la recorrida virtual por segmentos y la detección de lesiones. Inmediatamente después los pacientes eran sometidos a colonoscopía convencional. Luego de la revisión ciega de cada segmento se revelaban los datos del segmento homólogo estudiado por colonoscopía virtual. En caso de disparidad de resultados se reexaminaba el segmento dado (colonoscopía no ciega). La combinación de ambos resultados (colonoscopía convencional ciega y no ciega) constituyó el test de referencia. Se realizó biopsia de todas las lesiones. Los pacientes completaron luego un cuestionario de satisfacción.

\section{Medición de Resultados Principales}

Punto final primario: sensibilidad de ambos métodos y especificidad de CV para la detección de lesiones de al menos $6 \mathrm{~mm}$ de diámetro. Puntos finales secundarios: detección de todas las lesiones, lesiones avanzadas (displasia, componente velloso), preferencias del paciente.
Resultados

Tabla 1: Características operativas de la CV vs CO

\begin{tabular}{|c|c|c|c|c|c|}
\hline \multirow{2}{*}{$\begin{array}{c}\text { Variable } \\
\text { Tamaño Pólipo }\end{array}$} & \multirow[b]{2}{*}{$>=6 \mathrm{~mm}$} & \multicolumn{2}{|c|}{$\begin{array}{c}\text { n/total n } \\
\text { (\%[95\%/C]) }\end{array}$} & \multirow[b]{2}{*}{$>=9 \mathrm{~mm}$} & \multirow[b]{2}{*}{$>/=10 \mathrm{~mm}$} \\
\hline & & $>/ / 7 \mathrm{~mm}$ & $>\mid=8 \mathrm{~mm}$ & & \\
\hline \multicolumn{6}{|c|}{ Colonoscopía virtual } \\
\hline Sensibilidad & $\begin{array}{c}149 / 168 \\
(88.7[82.9-93.1])\end{array}$ & $\begin{array}{c}100 / 110 \\
(90.9[83.9-95.6])\end{array}$ & $\begin{array}{c}77 / 82 \\
(93.9[86.3-98.0])\end{array}$ & $\begin{array}{c}53 / 57 \\
(93.0[83.0-98.1])\end{array}$ & $\begin{array}{c}45 / 48 \\
(93.8[82.8-98.7])\end{array}$ \\
\hline Especificidad & $\begin{array}{c}848 / 1065 \\
(79.6[77.0-82.0])\end{array}$ & $\begin{array}{c}981 / 1123 \\
(87.4[85.3-89.2])\end{array}$ & $\begin{array}{c}1061 / 1151 \\
(92.2[90.5-93.7])\end{array}$ & \begin{tabular}{|c|}
$1116 / 1176$ \\
$(94.9[93.5-96.1])$
\end{tabular} & $\begin{array}{c}1138 / 1185 \\
\text { (96.0[94.8-97.1]) }\end{array}$ \\
\hline TVP & $\begin{array}{c}366 / 1233 \\
(29.7[27.1-32.3])\end{array}$ & $\begin{array}{c}242 / 1233 \\
(199.6[17.4-22.0])\end{array}$ & $\begin{array}{c}167 / 11233 \\
(13.5[11.7-15.6])\end{array}$ & $\begin{array}{c}113 / 1233 \\
(9.2[7.6-10.9]) \\
\end{array}$ & $\begin{array}{c}92 / 1233 \\
(7.5[6.1-9.1])\end{array}$ \\
\hline \multicolumn{6}{|c|}{ Colonoscopía óptica inicial } \\
\hline Sensibilidad & $\begin{array}{c}155 / 168 \\
(92.3[87.1-95.8))\end{array}$ & $\begin{array}{c}100 / 110 \\
(90.9[83.9-95.61)\end{array}$ & $\begin{array}{c}75 / 82 \\
(91.583 .2-96.5) \mid\end{array}$ & $\begin{array}{c}51 / 57 \\
(89.5778 .5-96.01)\end{array}$ & $\begin{array}{c}42 / 48 \\
(87.5[74.8-95.3])\end{array}$ \\
\hline
\end{tabular}

*TVP: tasa verdaderos positivos. IC: intervalo de confianza

La prevalencia de pólipos adenomatosos fue del 13.6\% ( 6-8 $\mathrm{mm}$ ), $6.7 \%(8-10 \mathrm{~mm}$ ) y de $3.9 \%$ (desde $10 \mathrm{~mm}$ ).

Se hallaron 2 pólipos adenomatosos $(0.4 \%)$ malignos, de los cuales 1 no fue diagnosticado por $\mathrm{CO}$.

En general, la sensibilidad de ambos métodos para la detección de pólipos fue similar, aunque la CV fue ligeramente superior para detectar pólipos mayores de $8 \mathrm{~mm}$ (diferencia no estadísticamente significativa).

El valor predictivo negativo de la colonoscopía virtual para pólipos mayores de $8 \mathrm{~mm}$ fue del $99 \%$.

La performance diagnóstica fue uniforme en todos los centros, con un adecuado acuerdo interobservador (k 0.75-0.8). *

El $54.3 \%$ de los pacientes refirió mayor incomodidad con la CV en relación a la CO (38\%). Aun así, el $68,3 \%$ la calificó como "más aceptable" en términos de conveniencia global, y más pacientes ( 49,8 vs $41,1 \%)$ expresaron su preferencia hacia la CV para un próximo screening.

\section{Conclusiones}

La exploración virtual del colon utilizando la reconstrucción tridimensional inicial mostró eficacia diagnóstica similar a la colonscopía convencional.

Fuente de financiamiento: Fondo para el avance de las prácticas médicas, Departamento de defensa, Gobierno de EEUU.

\section{Comentario}

Este artículo constituye uno más dentro de los ya publicados en la búsqueda de un método alternativo con buenas características operativas para la detección precoz del cáncer de colon, y que logre incrementar la baja aceptación de los métodos invasivos de rastreo, a pesar de la comprobada disminución de la mortalidad ${ }^{1}$. Si bien este trabajo muestra un buen rendimiento diagnóstico para la $\mathrm{CV}$, es de destacar que hasta la fecha existe gran disparidad en los resultados reportados. Así, un estudio recientemente publicado en el JAMA ${ }^{2}$ muestra sensibilidades de entre $39-55 \%$ para pólipos de 6 a $10 \mathrm{~mm}$ respectivamente.

En el presente estudio se utiliza la modalidad de confrontación por segmentos de colon estudiados, común a la mayoría de protocolos diagnósticos de cáncer colónico, aunque con características distintivas: una mayor discriminación de la imagen endoluminal, la evaluación inicial utilizando la reconstrucción tridimensional, tomógrafos de alta resolución y tomografistas con buen grado de entrenamiento (habían realizado como mínimo 25 estudios previos y algunos mas de 100).

Entre los beneficios que aportaría el screening de cáncer de colon utilizando la CV se encuentran una menor duración del procedimiento y la no utilización de anestesia general. Aunque el número de pacientes es insuficiente para evaluar el riesgo de perforación colónica, éste podría ser menor que el de la $\mathrm{CO}$.

Entre las desventajas, la CV no permite la toma de biopsia, con lo cual de acuerdo al tamaño del pólipo hallado, algunos pacientes deberían complementar el estudio con la $\mathrm{CO}$ ( 1 de 3 para pólipos de $6 \mathrm{~mm}$; 1 de 7 para $8 \mathrm{~mm}$; y 1 de 13 para $10 \mathrm{~mm}$ ). También el mayor costo, la falta de disponibilidad de tomógrafos de alta resolución en la mayoría de los centros, y la carencia de tomografistas adecuadamente entrenados son limitaciones de importante peso en países como el nuestro.

En cuanto al grado de aceptación por los pacientes, el resultado también es variable y se encuentra influenciado por la información que se brinde previamente, si bien en este estudio se manifestó una tendencia favorable hacia la colonoscopía virtual.

\section{Conclusiones del comentador}

La colonoscopía virtual es una herramienta promisoria en el mediano plazo para el rastreo de cáncer de colon, aunque faltan más estudios con diseño similar que permitan corroborar la buena precisión diagnóstica reportada en el presente trabajo y que evalúen su costo-efectividad

\section{Dr. Gabriel Villalón [Unidad de Medicina Familiar y Preventiva. Hospital Italiano de Buenos Aires]}

* ver glosario

Villalón G. La colonoscopía virtual podría ser eficaz para el rastreo de cáncer de colon. Evid. actual. práct. ambul. 2004;7:108. Comentado de: Pickhardt PJ, Choi JR, Hwang I, y col. Computed Tomographic Virtual Colonoscopy to Screen for Colorectal Neoplasia in Asymptomatic Adults. NEJM 2003;349:2191-200

\section{Bibliografía}

1.Kopitowski K. Problemas frecuentes en coloproctología. En: Rubinstein E. y col.: PROFAM. $2^{a}$ ed. Bs As; 2001.

2.Cotton PB et al. Computed Tomographic Colonography, a multicenter comparison with standard colonoscopy for detection of colorectal neoplasia. JAMA 2004; 291: 1713-19. 